\title{
A PHASE 3B, OPEN-LABEL, EXTENSION STUDY TO EVALUATE THE LONG-TERM SAFETY OF ONCE-DAILY DUAL-RELEASE HYDROCORTISONE IN PATIENTS WITH ADRENAL INSUFFICIENCY
}

\section{A Nilsson,' R Bergthorsdottir,' P Burman, ${ }^{2}$ P Dahlqvist, ${ }^{3}$ B Ekman,, BE Engström, ${ }^{5}$ O Ragnarsson,' S Skrtic, ${ }^{1,6}$ JWahlberg, ${ }^{4}$ H Achenbach, ${ }^{7}$ S Uddin, ${ }^{8}$ C Marelli, ${ }^{7}$ G Johannsson'}

I. Department of Endocrinology, Sahlgrenska University Hospital and Institute of Medicine, Sahlgrenska Academy, University of Gothenburg, Gothenburg, Sweden; 2. Department of Endocrinology, Skåne University Hospital Malmö, University of Lund, Lund, Sweden; 3. Department of Public Health and Clinical Medicine, Umeå University, Umeå, Sweden; 4. Department of Endocrinology and Department of Medical and Health Sciences, Linköping University, Linköping, Sweden; 5. Department of Medical Sciences, Endocrinology and Mineral Metabolism, University Hospital, Uppsala, Sweden; 6. AstraZeneca R\&D, Mölndal, Sweden; 7. Shire International GmbH, Zug, Switzerland; 8. Shire, Lexington, Massachusetts, USA

\section{INTRODUCTION}

Patients with adrenal insufficiency (Al) require lifelong glucocorticoid replacement therapy. Standard treatments are unable to replicate the daily physiological cortisol profile, potentially leading to adverse metabolic, cardiovascular and sleep consequences.

In addition, quality of life $(\mathrm{Q} o \mathrm{~L})$ continues to decline over time with conventional treatment in patients with $\mathrm{Al} .5$

The recently introduced once-daily (OD), dual-release hydrocortison (DR-HC) tablet has demonstrated a more physiological cortiso

DR-HC therapy has been shown to significantly improve cardio-

metabolic factors and QoL compared with conventional treatment. . $^{5-7}$

- To investigate the long-term safety and tolerability of DR-HC OD
in patients with primary AI.

\section{METHODS}

This was an open-label, multicentre, long-term extension study in adult patients with primary Al receiving stable glucocorticoid

eplacement therapy (Figure I).

Patients received oral DR-HC each morning in the fasted state, using the same total daily dose of hydrocortisone as they were receiving at

Dose could be adjusted according to the treating physician's judgement.

Outcomes

Safety and tolerability outcomes included adverse events (AEs), intercurrent illness episodes, increased hydrocortisone need, laboratory parameters, vital signs, and results of patient/investigator questionnaires.

QoL was evaluated using the Fatigue Impact Scale (FIS) and the Psychological General Well-Being (PGWB) questionnaire. Statistical analyses

Aill statistical analyses were performed on the safe

Subgroup analyses included patients recruited from the randomized

crossover plus 6-month extension study, newly recruited patients and patients with diabetes mellitus (DM). DM was defined as DM

during the 5 -year extension study.

Exploratory analyses evaluated change over time using the Wilcoxo signed rank test

\section{RESULTS}

Baseline demographics and disease characteristics

Seventy-one patients entered the 5-year open-label extension study

and were included in the safety population (Table I).

Long-term safety

In the 5-year extension
327.7 patient-years.

327.7 patient-years.
Seventy patients $(98.6 \%$

100 patient-years).

AE frequency was higher in newly recruited patients versus

patients recruited from the randomized crossover plus 6-month

extension study (528.9 vs 273. I per 100 patient-years).

100 patient-years) and elderly patients (268.7 per 100 patient-

years) versus the overall safety population.

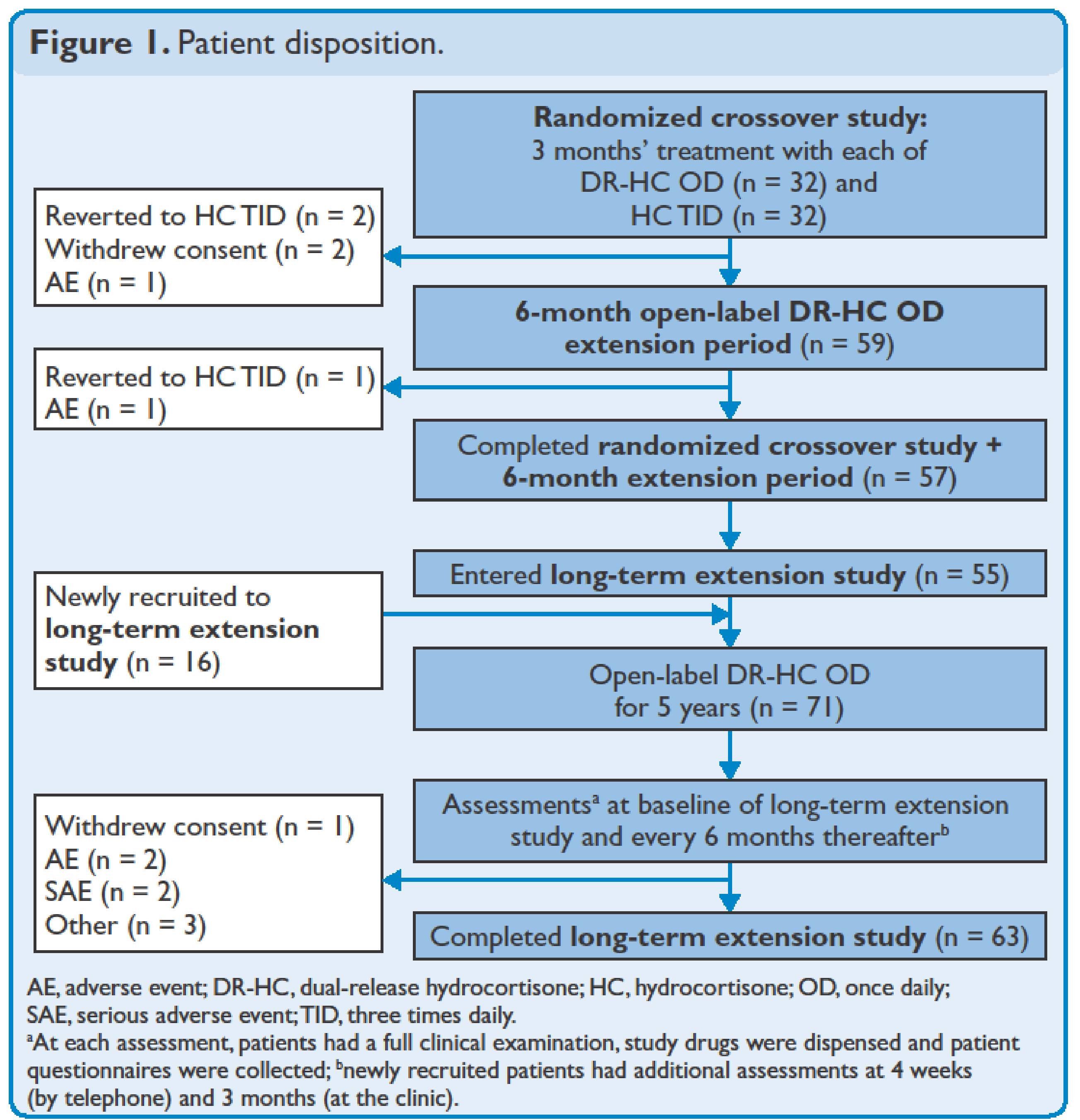

\begin{tabular}{|c|c|c|c|c|}
\hline \multirow{2}{*}{ Iable I. Der } & \multirow[t]{2}{*}{$\begin{array}{c}\text { All patients } \\
(n=7 I)\end{array}$} & \multicolumn{2}{|c|}{$\begin{array}{l}\text { Subgroups by } \\
\text { recruitment status }\end{array}$} & \multirow{2}{*}{$\begin{array}{c}\text { Patients } \\
\text { with DMM } \\
(\mathrm{n}=14)\end{array}$} \\
\hline & & \begin{tabular}{|c|}
$\begin{array}{c}\text { From } \\
\text { previous study } \\
(\mathbf{n}=55)\end{array}$ \\
\end{tabular} & $\begin{array}{c}\text { Newly } \\
\text { recruited } \\
(\mathrm{n}=16)\end{array}$ & \\
\hline Age (years) & $48.2(13.3)$ & $49.0(13.4)$ & $45.6(13.1)$ & $56.2(12.8)$ \\
\hline \multicolumn{5}{|l|}{ Sex } \\
\hline $\begin{array}{l}\text { Male } \\
\text { Female }\end{array}$ & $\begin{array}{l}36(50.7 \%) \\
35(49.3 \%)\end{array}$ & $\begin{array}{l}31(56.4 \%) \\
24(43.6 \%)\end{array}$ & $\begin{array}{l}5(31.3 \%) \\
11(68.8 \%)\end{array}$ & $\begin{array}{l}8(57.1 \%) \\
6(42.9 \%)\end{array}$ \\
\hline BMI $\left(\mathrm{kg} / \mathrm{m}^{2}\right)$ & $25.5(4.1)$ & $25.8(3.8)$ & $24.5(4.8)$ & $28.2(4.7)$ \\
\hline $\begin{array}{l}\text { Duration of Al } \\
\text { (years) }\end{array}$ & $16.7(11.0)$ & $17.5(11.0)$ & $14.0(11.0)$ & $20.3(12.7)$ \\
\hline $\begin{array}{l}\text { Diabetes } \\
\text { mellitus }\end{array}$ & $14(19.7 \%)$ & II (20.0\%) & $3(18.8 \%)$ & $14(100.0 \%)$ \\
\hline Hypertension & $16(22.5 \%)$ & $14(25.5 \%)$ & $2(12.5 \%)$ & $7(50.0 \%)$ \\
\hline
\end{tabular}

The most common AEs were nasopharyngitis (70.4\%), fatigue (52.1\%) and gastroenteritis (47.9\%); most AEs (84.8\%) were unrelated to DR-HC. years); four were possibly related to DR-HC: acute Al $(\mathrm{n}=2)$, gastritis $(n=1)$ and syncope $(n=1)$.

Tro deaths were reported (fall from height subarachnoid

Intercurrent illness and increased hydrocortisone use Over the 5-year study, there were 709 episodes of intercurrent use not due to intercurrent illness in 43 patients (Table 2).

Cardiometabolic parameters and vital signs

Small but significant increases from baseline in the 5-year extensio study were observed at all time points for mean fasting plasma (Figure 2b) in the overall safety population.

Mean HDL-cholesterol was significantly increased at all time points in the overall safety population and at 36 and 60 months in patients with DM (Figure 3). Other lipid parameters were similar at baseline and 5 years in the overall safety population and in patients with DM. There were no statistically significant changes in vital signs, body

weight or body mass index.

Table 2. Episodes of intercurrent illness and increased hydrocortisone use not due to intercurrent illness, over time.

\begin{tabular}{c|c|c|c|c|c}
$\begin{array}{c}0-12 \\
\text { months } \\
(\mathrm{n}=71)\end{array}$ & $\begin{array}{c}12-24 \\
\text { months } \\
(\mathrm{n}=68)\end{array}$ & $\begin{array}{c}24-36 \\
\text { months } \\
(\mathrm{n}=68)\end{array}$ & $\begin{array}{c}36-48 \\
\text { months } \\
(\mathrm{n}=67)\end{array}$ & $\begin{array}{c}48-60 \\
\text { months } \\
(\mathrm{n}=67)\end{array}$ & $\begin{array}{c}0-60 \\
\text { months } \\
(\mathrm{n}=71)\end{array}$ \\
\hline
\end{tabular}

\begin{tabular}{|c|c|c|c|c|c|c|}
\hline \multicolumn{7}{|c|}{ Intercurrent illness } \\
\hline $\begin{array}{l}\text { Episodes per } \\
\text { patient }\end{array}$ & $\begin{array}{l}2.93 \\
(3.86) \\
\end{array}$ & $\begin{array}{r}3.38 \\
(5.05) \\
\end{array}$ & $\begin{array}{c}5.42 \\
(16.73) \\
\end{array}$ & $\begin{array}{r}3.83 \\
(9.63) \\
\end{array}$ & $\begin{array}{c}2.64 \\
(3.41)\end{array}$ & $\begin{array}{l}11.08 \\
(23.81)\end{array}$ \\
\hline $\begin{array}{l}\text { Days per } \\
\text { episode }\end{array}$ & $\begin{array}{l}6.52 \\
(19.70) \\
\end{array}$ & $\begin{array}{l}8.26 \\
(19.07) \\
\end{array}$ & $\begin{array}{l}3.78 \\
(2.76) \\
\end{array}$ & $\begin{array}{c}9.10 \\
(28.40) \\
\end{array}$ & $\begin{array}{r}2.60 \\
(2.64) \\
\end{array}$ & $\begin{array}{l}4.70 \\
(8.16) \\
\end{array}$ \\
\hline $\begin{array}{l}\text { Dose per } \\
\text { episode (mg) }\end{array}$ & $\begin{array}{l}19.16 \\
(11.39)\end{array}$ & $\begin{array}{l}18.92 \\
(12.13)\end{array}$ & $\begin{array}{r}23.47 \\
(13.87)\end{array}$ & $\begin{array}{l}18.65 \\
(8.88) \\
\end{array}$ & $\begin{array}{l}19.12 \\
(13.07)\end{array}$ & $\begin{array}{r}20.48 \\
(11.15) \\
\end{array}$ \\
\hline \multicolumn{7}{|c|}{ Increased hydrocortisone use not due to intercurrent illness } \\
\hline $\begin{array}{l}\text { Episodes per } \\
\text { patient }\end{array}$ & $\begin{array}{l}6.15 \\
(8.72) \\
\end{array}$ & $\begin{array}{r}10.00 \\
(14.97) \\
\end{array}$ & $\begin{array}{l}7.57 \\
(9.11) \\
\end{array}$ & $\begin{array}{r}10.43 \\
(16.31) \\
\end{array}$ & $\begin{array}{r}5.37 \\
(7.69) \\
\end{array}$ & $\begin{array}{r}22.88 \\
(33.53) \\
\end{array}$ \\
\hline $\begin{array}{l}\text { Days per } \\
\text { episode }\end{array}$ & $\begin{array}{r}2.11 \\
(2.06) \\
\end{array}$ & $\begin{array}{l}1.83 \\
(1.27) \\
\end{array}$ & $\begin{array}{l}3.37 \\
(6.57)\end{array}$ & $\begin{array}{c}4.56 \\
(10.90) \\
\end{array}$ & $\begin{array}{c}5.34 \\
(14.14) \\
\end{array}$ & $\begin{array}{l}2.15 \\
(1.58) \\
\end{array}$ \\
\hline $\begin{array}{l}\text { Dose per } \\
\text { episode }(\mathrm{mg})\end{array}$ & $\begin{array}{l}15.26 \\
(10.52)\end{array}$ & $\begin{array}{l}15.49 \\
(14.99)\end{array}$ & $\begin{array}{l}14.62 \\
(12.33)\end{array}$ & $\begin{array}{l}13.36 \\
(9.57)\end{array}$ & $\begin{array}{l}14.53 \\
(10.33)\end{array}$ & $\begin{array}{l}15.48 \\
(9.60)\end{array}$ \\
\hline
\end{tabular}

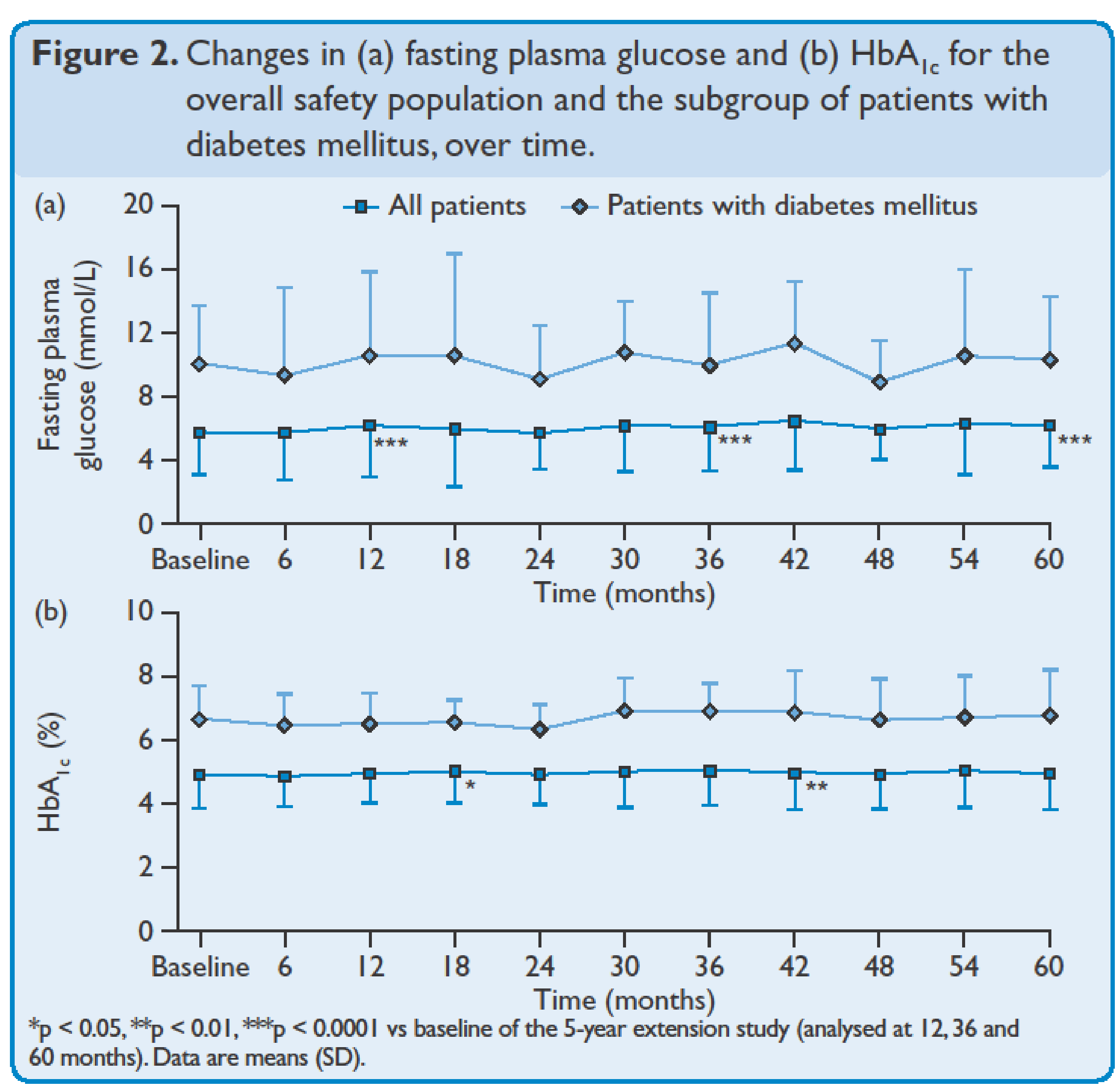

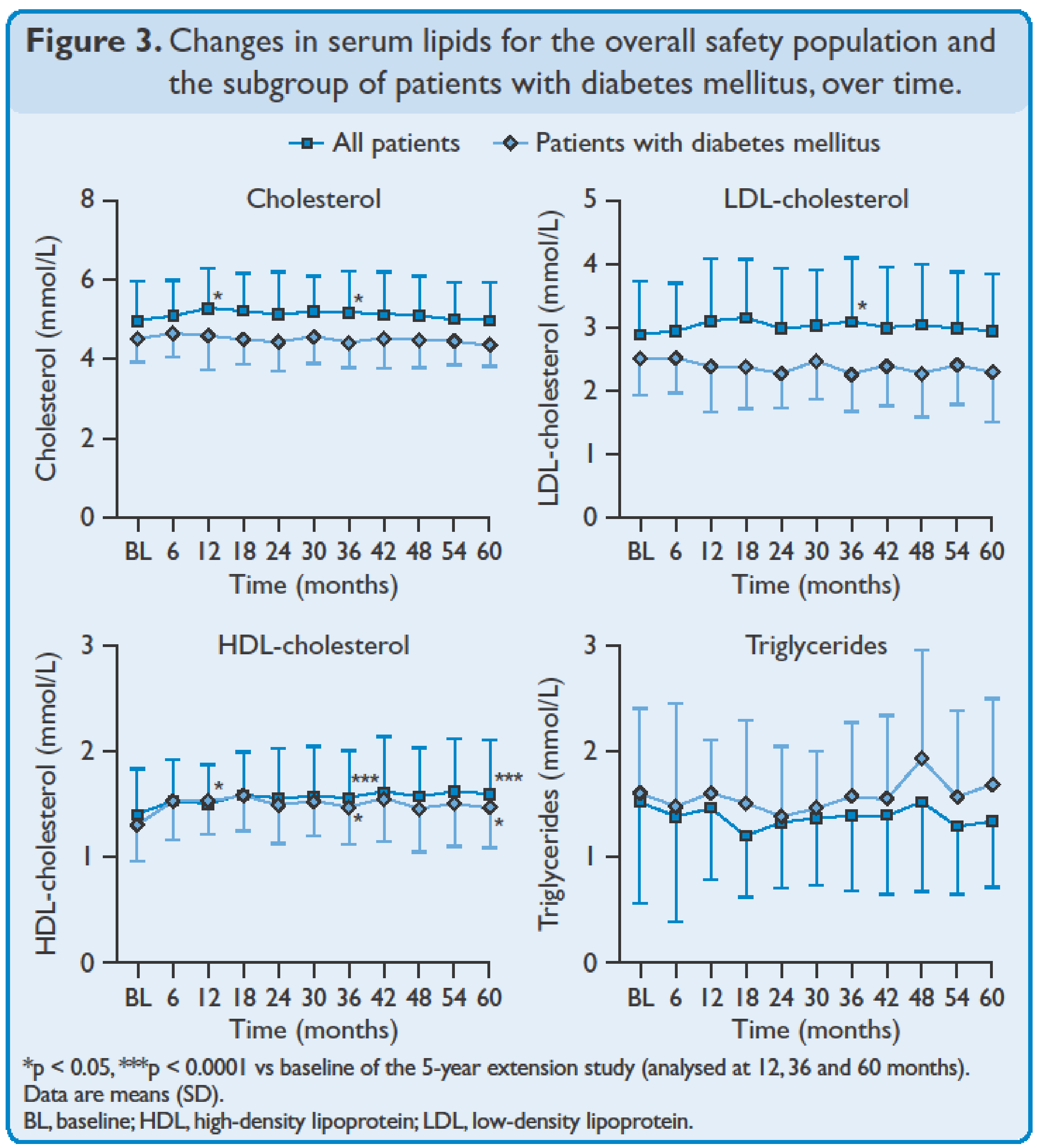

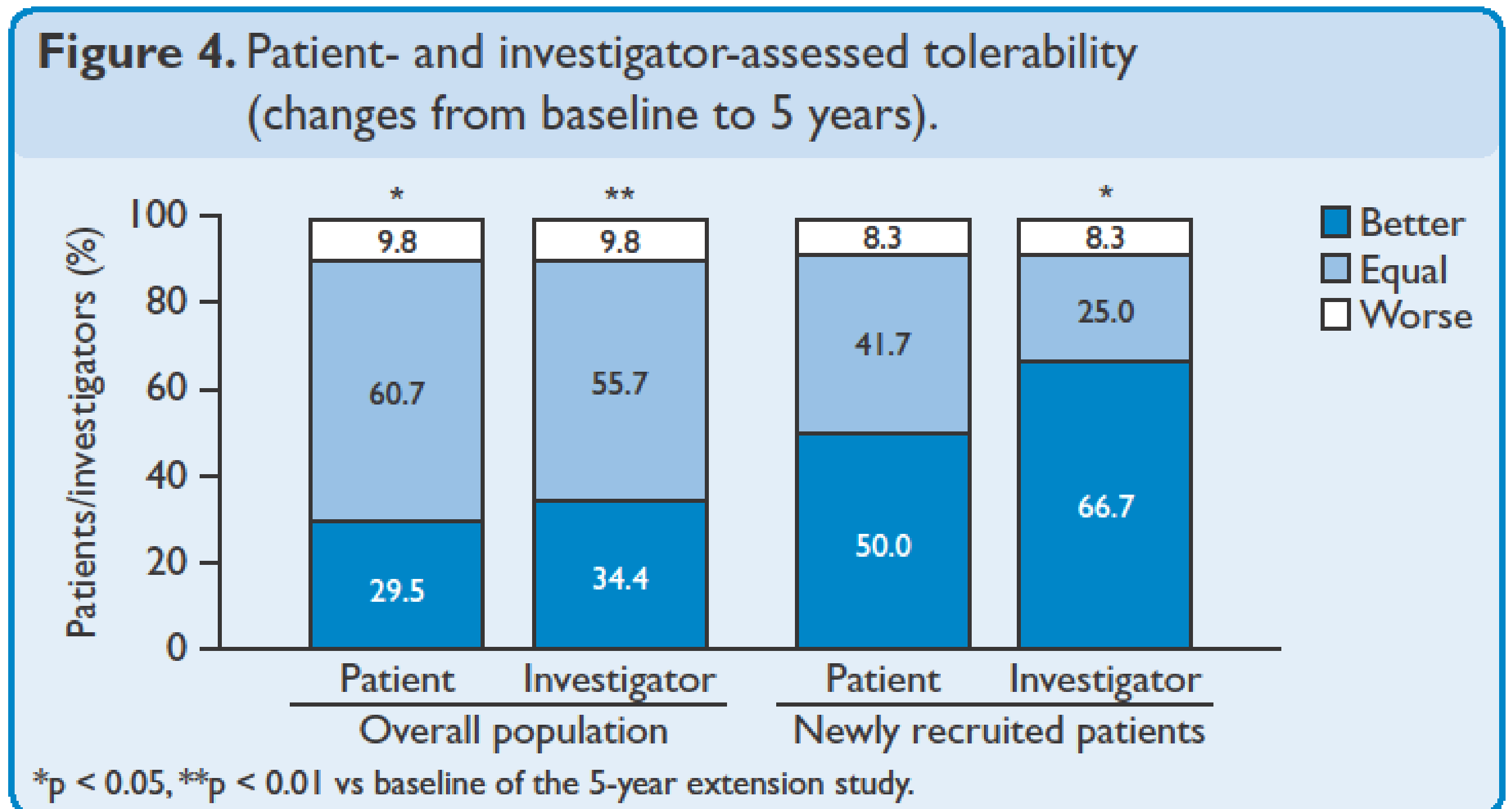

Patient- and investigator-assessed tolerability$$
\text { Patient- and investigator-assessed treatment tolerability was }
$$

QoL

There were no significant changes in FIS or PGWB total scores from baseline to 5 years. However, FIS physical functioning was significantly PGWB scores numerically improved after 5 years.

\section{CONCLUSIONS}

Long-term data demonstrate that the newly developed treatment option, DR-HC, is well tolerated with no safety concerns in patients with primary Al, including those with DM. The number of intercurrent illness episodes was similar for DR-HC and hydrocortisone three times daily in the randomized crossover plus 6-month extension study, , while intercurrent illness over 5 years' DR-HC treatment.

Patient- and investigator-assessed tolerability was significantly improved with long-term DR-HC treatment compared with baseline. The worsening of physical functioning over time reported here may
reflect the decline in QoL commonly seen with ageing and with chronic diseases. ${ }^{9}$

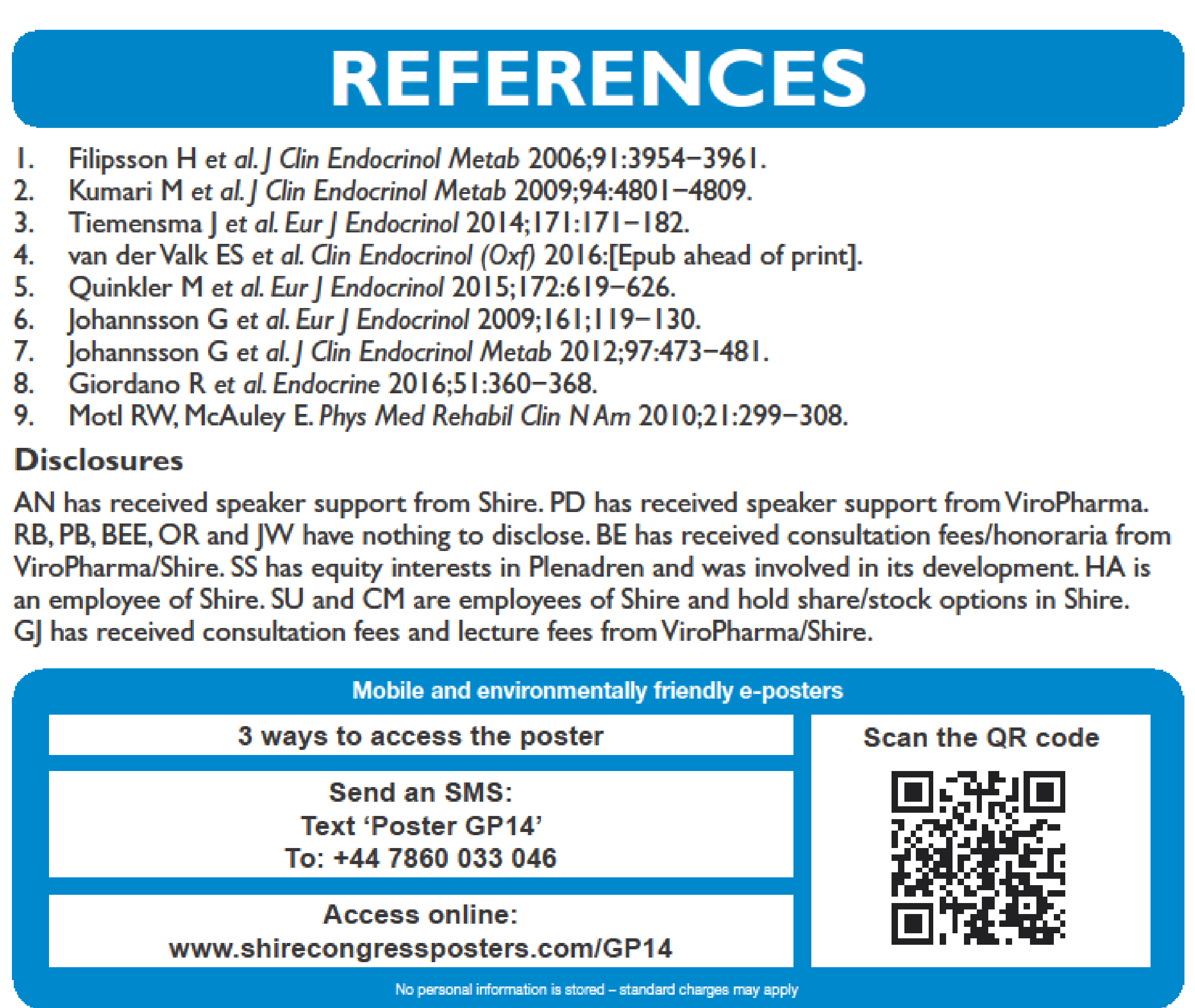

